MummY: AAtTTCCCETGA CAACTTT GAAGCTTTTCGTAT 33 da-7 : AAATTTCCCTGAACAACTTTTGAAGCTTTTTCGTAT 348 GTCTCCTGTAGTAGATCTTGGGGTCGTTCCATCAATTATAT 74 GTCTCCTGTAGTAGATCTTGGGGTCGTTCCATCAATTATAT 307 ACTCTATAGATATTAAAAA GTTGCCCGTTTCTTTCTCTCA 114 ACTCTATAGATATTAAAAAAGTTGCCCGTTTCTTTCTCTCA 266 GACTTACTCACATTTCCACATGGGAACTGGCACAGGTGGGG 155 GACTTACTCACATTTCCACATGGGAACTGGCACAGGTGGG 225

AGTGGGTAAAGGAGTCCAGCAGGTCGAATGCCITCAACAAT 196 AGTGGGTAAAGGAGTCCAGCAGGTCGAATGCCTTCAACAAT 184

CATTTTACCACATGGTCCTCACTTACTCTCAGCTGCCTCAT 237 CATTTTACCACATGGTCCTCACTTACTCTCAGCTGCCTCAT 143

ATGTGTCACCTCACAAA TAATCAAATAAAA TGGGCATG 275 ATGTGTCACCTCACAAAATAATCAAAATAAAAATGGGCATG 102

IAGCTAAGCTTTGTAAATAGTGAAAACATGGATGTCAATTG 316 TAGCTAAGCTTTGTAAATAGTGAAAACATGGATGTCAATTG 61

TTTTTACATATTTCTATTACAGGTATAGCTTCACATTITTC 357 TTMTACATATTTCTATTACAGGTATAGCTTCACATTTITC 20

MTAGCAAAATAAggGaTC

TTTAGCAAAATAAGGGATC

FIG. 2 Comparison of mummy DNA clone pMUM2:9 and dq-7. Lower-case letters mark non-identitical bases; a few gaps have been introduced to maximize the alignment.

element, which turns out to be an $A l u$ repeat, having $\sim 80 \%$ identity with the Alu family average sequence and $~ 70 \%$ identity with the $A l u$ member present in pMUM2:9 (nucleotides 494-782). Surprisingly, there is $97 \%$ identity between nucleotides 1-382 of dq-7 and nucleotides 1-376 of pMUM2:9 (Fig. 2), which excludes the $A l u$-like sequence of the latter. The sequence in the HLA-DQA2 gene equivalent to nucleotides 1-382 of dq-7 has about $87 \%$ identity to nucleotides 1-376 of pMUM2:9. Quantitative hybridization assays (calibrated with $A l u$ and Ll probes) and an exhaustive search in the computer catalogue (Microgenie, Beckman, 1987) demonstrate that the sequence is not a previously undescribed repetitive element.

One explanation of these findings may lie in the fact that pMUM2:9 was selected as a repetitive DNA-containing sequence by virtue of its hybridization with a $B g I I /$ SphI genomic DNA fragment ${ }^{1}$ that was derived from a HLA-DR $\beta$ pseudogene ${ }^{4}$. This fragment, however, does not contain sequences (other than $\mathrm{Alu}$ ) similar to either pMUM2:9 or to dq-7. Nevertheless, the $B g l I I / S p h I$ probe may encompass a sequence characteristic of MHC class II genes that made possible the inadvertent isolation of HLA-DQA gene-related sequences from mummy DNA.

The high degree of conservation of a rather extended region of the first introns of the HLA-DQA1 gene of an ancient Egyptian individual and the DR4.4 cell line $^{2}$ strikingly contrasts with the frequency of polymorphism observed in modern population studies.

GIOVANNA DEL POZZO JOHN GUARDIOIA

The International Institute of Genetics and Biophysics,

CNR, 1-80125 Naples, Italy

1. Pääbo, S. Nature 314, 644-645 (1985).

2 Okada, K et al. Proc natn. Acad Sci. U.S.A. 82, 3410 (1985).

3. Auffray, C. etal. Immunogenetics 26, 63-73 (1987)

4. Larhammar, D.et al. Proc. natn. Acad. Sci. U.S.A. 82 1475 (1985).

\section{Female choice}

SIR-von Schantz et al. ${ }^{1}$ report that female pheasants (Phasianus colchicus) choose males on the basis of the length of the male's spur in preference to other biometric variables or territory quality. They find no correlation between the quality of a male's territory and the number of females settling on it. This, however, does not necessarily mean that females do not assess and choose males on the basis of their territories; perhaps the cues females use have not been found or adequately measured.

von Schantz et al. base their conclusion on the assumption that their measure of territory quality is in fact what females are using to assess territories. To support this assumption, they demonstrate a statistically significant correlation between their index of territory quality and the number of chicks hatched. Their correlation coeffient is rather low $(r=0.31)$, however, which means that less than 10 per cent of the variance in chick number is explained by territory quality ${ }^{2}$, suggesting their quality index is unreliable.

A second important point to their argument is an absence of a relationship between spur length and dominance in captive birds during the winter. Because male pheasants typically form winter flocks and then set up solitary territories during the breeding season ${ }^{3}$, the degree and nature of intrasexual competition is likely to be very different in the two seasons. It seems a great leap of faith to assume that dominance relationships of captive birds in one season will be an accurate predictor of their territory quality obtained when free living in the next season. Indeed, the only dominance reversals seen in one study ${ }^{3}$ occurred at the time that males were setting up territories. von Schantz et al. tested their findings

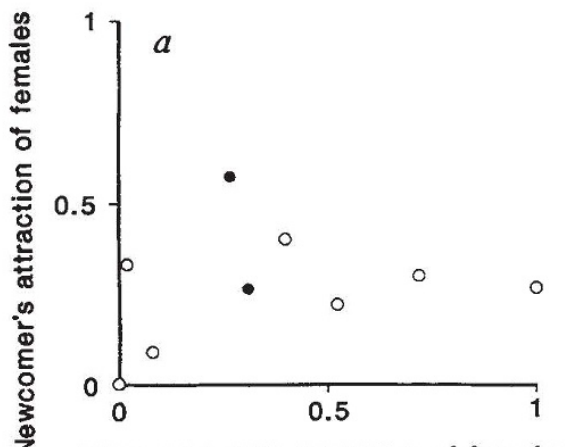

Former owner's attraction of females further by manipulating spur length, and find that long-spurred males have more females but are not more dominant. But again they fail to measure dominance during the critical time when territories are being established and females are choosing mates, and they do not assess the males' territories at all. It is hard to believe that spurs do not function in intra-sexual selection, because males use them for intrasexual combat when setting up territories ${ }^{4}$.

An alternative explanation for their results is that females are choosing males indirectly by their territories because territory quality has at least some effect on their reproductive success. Males, on the other hand, use their spurs in combat to obtain the best territories. This would also best explain the finding that females mating with long-spurred males rear the greatest number of chicks, because these females would be in the best areas. Given that spurs are used in combat, that territory quality is important to female reproductive success, and that female choice for territory quality is well known in other birds $^{5}$, this hypothesis seems more likely.

Udo M. SaVAlLI

Department of Zoology,

University of California,

Berkeley, California 94720, USA

VON SCHANTZ ET AL. REPLY-In our study', we measured male dominance in the enclosure during March - the birds' last month in captivity. On the first week-day of April all birds were released and less than 2 weeks later most males had established territories (median $=9$ days after release, data from $1984-87, n=93$ ). We believe that dominance measured in this way gives accurate data about the outcome of intrasexual conflicts uncorrelated to ownership of a given territory. This is important because we aimed to study the effects of male characteristics on male

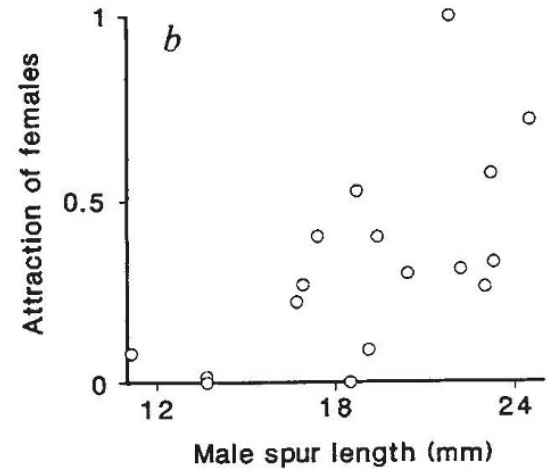

a. Male attraction of females (measured as in Tables 1 and 3 in ref. 1 ) as a function of the former territory owner's attractiveness $(r=0.23, P=0.56, n=9)$. The sample is based on 9 cases, including 17 pheasant males, where the same territory was occupied by different males during successive breeding seasons. A territory is considered to be the same between 2 years if the overlap of the two estimated territories, as measured by the harmonic mean method ${ }^{7}$, is $>70 \%$ of their average area. One territory was occupied by three different males during three consecutive breeding seasons and is therefore represented twice in the sample (filled circles). Attraction of females is presented as the proportion of the highest estimate recorded during the particular year (1986-88). $b$, Male attraction of females as a function of the 17 territory owners' spur length $(r=0.63, P=0.007, n=17)$. 